\title{
A Performance Analysis of the Network Formation Process in IEEE 802.15.4e TSCH Wireless Sensor/Actuator Networks
}

\author{
Domenico De Guglielmo, Alessio Seghetti, Giuseppe Anastasi \\ Dept. of Information Engineering \\ University of Pisa, Italy \\ E-mail: \{firstname.lastname\}@ iet.unipi.it
}

\author{
Marco Conti \\ Institute for Informatics and Telematics \\ National Research Council, Italy \\ E-mail: marco.conti@iit.cnr.it
}

\begin{abstract}
Time Slotted Channel Hopping (TSCH) is one of the access behavior modes defined in the IEEE 802.15.4e standard. It combines time slotted access with multi-channel and channel hopping capabilities, providing predictable latency, energy efficiency, high network capacity, and high communication reliability. In this paper we focus on the formation process of TSCH networks, which relies on the regular advertisement of Enhanced Beacons (EBs). We consider a simple random-based advertisement algorithm, and evaluate its performance, through analysis and simulation, in terms of joining time (i.e., total time taken by a new node to join the network). We found that the joining time depends on a number of factors and, mainly, on the number of channels used for $\mathrm{EB}$ advertisement.
\end{abstract}

Keywords: IEEE 802.15.4e, TSCH, network formation, performance evaluation.

\section{INTRODUCTION}

Wireless Sensor and Actuator Networks (WSANs) are expected to play a key role in the realization of the future Internet of Things that will connect to the Internet any kind of devices, living beings, and objects [1]. A WSAN consists of a number of sensor and actuator devices deployed over a geographical area and interconnected through wireless links. Sensor devices gather information from the physical environment (e.g., temperature, pressure, vibrations), perform a preliminary local processing, and send (raw or processed) data to a controller. Based on the received data, the controller takes intelligent decisions and performs appropriate actions, through actuator devices, to change the behavior of the physical environment [2]. WSANs are already used in many application domains, ranging from traditional environmental monitoring and location/tracking applications to industrial [3] and healthcare [4] applications. In the industrial field WSAN applications include factory automation [5], distributed and process control [6][7][8], real-time monitoring of machinery health, detection of liquid/gas leakage, radiation check [9], and so on. In the healthcare domain WSANs have been considered for the monitoring of physiological data in chronicle patients and transparent interaction with the healthcare system [10][11].

In recent years, many standards have been issued by international bodies to support the development of WSANs in different application domains. They include IEEE 802.15.4 [12], ZigBee [13], Bluetooth [14], WirelessHART [15] and ISA-100.11a [16]. In 2012 IEEE released the 802.15.4e standard [17] that extends the original 802.15.4 standard to address the emerging needs of embedded industrial applications. IEEE 802.15.4e defines a number of MAC (Medium Access Control) behavior modes, to support specific application domains, and some general functional improvements not tied to any specific application domain. In this paper we focus on the Time Slotted Channel Hopping (TSCH) mode [17], which combines time slotted access - already available in the original 802.15.4 MAC protocol - with multi-channel and channel hopping capabilities, thus providing increased network capacity, high reliability and predictable latency, while maintaining very low duty cycles (i.e., energy efficiency). The TSCH mode is topology-independent as it can be used to form any network topology (e.g., star, tree, partial mesh or full mesh).

So far, researches on $802.15 .4 \mathrm{e}$ TSCH have focused mainly on link scheduling, i.e., on how to assign links to nodes for data transmissions. A number of link scheduling algorithms have been proposed in the literature, that take either a centralized [18] or distributed approach [19]. Interactions between TSCH and protocols defined for the Internet of Things have been also addressed [20]. In this paper we focus on the TSCH network formation process. This process is strongly influenced by the policy used to announce the network presence, which is based on sending special messages named Enhanced Beacons (EBs). However, the standard does not specify any advertising policy as it is responsibility of a layer above the MAC.

In this paper we define a random-based advertisement algorithm that is a generalization of the algorithm used in [20]. We also analyze the considered algorithm in terms of joining time, i.e., total time taken by a device to join the network. Since a device has to keep the radio always on, while connecting to the network, the joining time is also a measure of the energy spent by the device to connect. To evaluate the performance of the considered advertisement algorithm we rely both on analysis and simulation, and investigate its sensitiveness to different parameters, such as number of used channel offsets, node density, packet error rate, and number of available frequencies. We found that the joining time is strongly influenced by the number of channels used for advertising EB messages. Using more channels reduces the joining time (and, hence, the energy spent to join the network). On the other side, using more channels for advertising increases the number of transmitted EBs. Hence, it reduces the bandwidth available for data transmissions and increases the energy spent for advertising the network. However, we found that, typically, 
using a large number of different channels is not necessary, unless the network density is extremely high.

The remainder of this paper is organized as follows. In Section II we introduce the 802.15.4e standard, while in Section III we focus on the TSCH mode and define our advertisement algorithm. In Section IV we model the network formation process through a discrete-time Markov chain. In Section V we show our analytical and simulation results. Finally, we draw conclusions in Section VI.

\section{IEEE 802.15.4e STANDARD}

The $802.15 .4 \mathrm{e}$ standard [17] extends the previous 802.15.4 standard [12] for low-rate, low-power, and lowcost Personal Area Networks (PANs), to address embedded industrial applications. To this purpose, it introduces two different categories of MAC enhancements, namely $M A C$ behaviors and general functional improvements. MAC behaviors are aimed to support specific applications, while general functional improvements are not tied to any specific application domain. Like in the original 802.15.4 standard, a PAN is formed by one PAN coordinator in charge of managing the whole network, and, optionally, one or more coordinators that are responsible for a subset of nodes in the network. Ordinary nodes must associate with a (PAN) coordinator in order to communicate, using a specific MAC behavior mode. The 802.1.5.4e standard [17] defines different MAC behavior modes (a detailed description is available in [21]). In this paper we focus on the Time Slotted Channel Hopping (TSCH) mode, which is the most general and complex one.

\section{TIME SLOTTED CHANNEL HOPPING}

TSCH [17] combines time slotted access with multichannel and channel hopping capabilities. Hence, it provides large network capacity, high reliability and predictable latency, while ensuring energy efficiency, thanks to the time slotted access mode. TSCH can be used with any network topology (e.g., star, tree, partial mesh or full mesh). However, it is particularly well-suited for multihop networks where multi-channel communication allows for an efficient use of the available resources.

\section{A. TSCH Access Mode}

In $\mathrm{TSCH}$ nodes synchronize on a periodic slotframe consisting of a number of timeslots. Figure 1 shows a slotframe with 5 timeslots. Each timeslot allows a node to send a maximum-size data frame and receive the related acknowledgement.

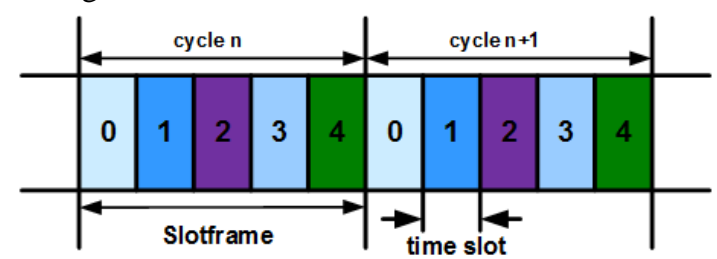

Fig. 1. Slotframe

If the acknowledgement is not received within a predefined timeout, the retransmission of the data frame is deferred to the next time slot assigned to the same (senderdestination) couple of nodes.

One of the main characteristics of $\mathrm{TSCH}$ is multichannel communication, based on channel hopping. In principle, 16 different channels can be used for communication, and each channel is identified by a channelOffset, i.e., an integer value in the range $[0,15]$. In practice, the number of available channels $\left(N_{c}\right)$ may be lower than 16 as some channels could be blacklisted, due to low communication quality. In TSCH a link is defined as the pairwise assignment of a directed communication between devices in a given timeslot on a certain channel offset [17]. Hence, a link between communicating devices can be represented by a couple specifying the timeslot in the slotframe and the channel offset used by the devices in that timeslot. Let $[n$, channeloffset $]$ denote a link between two devices. Then, the frequency $f$ to be used for communication in timeslot $n$ of the slotframe is derived as:

$$
f=F\left[(\text { ASN }+ \text { channelOff set }) \bmod N_{c}\right]
$$

where mod indicates the modulo operation while $A S N$ is the Absolute Slot Number, defined as the total number of timeslots elapsed since the start of the network (or an arbitrary start time determined by the PAN coordinator). It increments globally in the network, at every timeslot, and is thus used by devices as a timeslot counter. Using the multi-channel mechanism, several simultaneous transmissions can take place in the same timeslot, provided that different transmissions use different channel offsets. Also, Equation (1) implements the channel hopping mechanism by returning a different frequency for the same link at different slotframes.

A key element in TSCH is the link scheduling algorithm, i.e., how to assign links to nodes for data transmissions. However, the $802.15 .4 \mathrm{e}$ does not specify any specific algorithm as it is assumed to be implemented by upper layers. The standard just defines mechanisms to execute a link schedule. A number of link scheduling algorithms have been proposed in the literature [18] [19].

\section{B. TSCH PAN Formation}

Like the original 802.15.4 standard [12], TSCH supports two classes of devices, namely Full Function Devices (FFDs) and Reduced Function Device (RFDs). FFDs implement all the functionalities defined in the standard and can act as network coordinators. Instead, RFDs implement only a subset of functionalities and cannot act as coordinators.

The network formation starts when a FFD, typically the PAN coordinator, advertises the network presence by sending Enhanced Beacons (EBs) at regular times. EB messages are special TSCH frames containing the following information.

- Synchronization information (allows new devices to synchronize to the network);

- Channel hopping information (allows new devices to learn the channel hopping sequence);

- Timeslot information (describes when to expect a frame transmission and when to send an acknowledgment); 
- Initial link and slotframe information (allows new devices to know: (i) when to listen for transmissions from the advertising device, and (ii) when to transmit to the advertising device).

A device wishing to join the network starts scanning for possible EB messages. As soon as it receives a valid EB message from an advertiser node, the MAC layer notifies the higher layer. The latter initializes the slotframe and links, by exploiting the information in the received EB message, and switches the device into TSCH mode. At this point the device is connected to the network. Then, the device typically goes through a procedure aimed to allocate communication resources (i.e., slotframes and links) to the joining device. This procedure may also include a security handshake to mutually authenticate the joining device, configure encryption keys, and configure routing information. The mechanism and rules for setting up communication resources and configure security and routing policies are not defined in the $802.15 .4 \mathrm{e}$ standard, as they are under the responsibility of the higher layers (e.g., the application or network layer). Once connected and configured appropriately, devices may send EB messages, on their turn, to announce the network presence.

The EB advertising policy is not part of the $802.15 .4 \mathrm{e}$ standard as it is under the responsibility of upper layers (e.g., the application layer). To define an advertising policy we need to decide how to advertise (i.e., which nodes should send EB messages) and when to advertise (i.e., the rate at which EB messages should be sent by advertiser nodes). The simplest idea is letting all (full function) devices, that have already joined the network, to advertise EB messages. As far as the advertising rate, there are two contrasting requirements. On one hand, the rate should be as high as possible to allow devices to join the network quickly and save energy (it may be worthwhile pointing out that, while waiting for a valid EB message, the joining device must kept the radio always on). On the other hand, sending EB messages too often consumes bandwidth and energy at advertiser nodes. Also, in a real setting the advertising rate should vary dynamically, depending on the operating conditions (e.g., node density, packet error rate, available energy). To the purposes of our analysis we define a simple random-based advertisement algorithm that is a generalization of the algorithm used in the $\mathrm{TSCH}$ implementation described in [20].

\section{Random-based Advertisemt Algorithm}

In the random-based advertisement algorithm considered here all FFDs that have already joined the network act as advertiser nodes and broadcast periodic EB messages to announce the network. Each node is assigned, through the link scheduling algorithm, a link in the slotframe given by [timeslot, channeloffset] for the transmission of EB messages. Timeslots devoted to EB advertisement repeat periodically, with a period $T_{E B}$. For any advertiser node, if $T_{E B}$ (expressed in number of slots) and the number of available channels $N_{c}$ are relatively prime, the translation function of the multi-hopping mechanism ensures that EB messages are transmitted on different channels in subsequent cycles, and, above all, all the available $N_{c}$ channels are used over $N_{c}$ cycles.

A collision occurs during the transmission of an EB message, in a certain link, if two or more (advertiser) nodes transmit simultaneously. To reduce the collision probability each advertiser node transmits its EB message, at a scheduled link, with a probability $p_{E B}$ (and refrains with probability $\left.1-p_{E B}\right)$. The appropriate $p_{E B}$ value is derived autonomously by each node, depending on the local operating conditions (i.e., number of neighbors), in such a way to minimize the collision probability. Hence, different nodes generally use different $p_{E B}$ values. In Section IV we derive the optimal value for $p_{E B}$.

\section{PERFORMANCE ANALYSIS}

In this section, we model the node connection process, based on the advertisement algorithm described in the previous section, through a discrete-time Markov chain, and derive the joining time, i.e., the total time taken by a node to connect to the network. We also derive the optimal $p_{E B}$ value to be used by an advertiser node. We consider a single node $u$, willing to join the network (throughout referred as the joining node) and assume that there are $N$ advertiser nodes from which node $u$ can potentially receive an EB message. For simplicity, we assume that the advertisement period $T_{E B}$ is the same for all the advertiser nodes $\left(T_{E B}\right.$ and the number of available channels $N_{C}$ are assumed to be relatively prime). In our analysis we consider a worst-case scenario where all the advertiser nodes send their EB messages in the same timeslot, and using the same channel offset, at each period. In addition, they all are neighbors of each other.

In the considered scenario a simultaneous transmission of two (or more) advertiser nodes always results in a collision. As mentioned in the previous section, to reduce the collision probability each advertiser node actually transmits EB messages with a probability $p_{E B}$ (and refrains with probability $1-p_{E B}$ ). Hence, a successful EB transmission occurs when (i) only one advertiser node transmits its EB message and all the remaining $N-1$ nodes refrain, and (ii) the transmitted EB message is received correctly by the joining node. Assuming a packet error rate $p_{E R R}$, the probability that the joining node receives a valid EB message can be expressed as:

$$
P_{\text {valid }}=\left(1-p_{E R R}\right) \cdot\left[N \cdot p_{E B} \cdot\left(1-p_{E B}\right)^{N-1}\right]
$$

In Equation (2), the second term gives the probability that one advertiser node (out of $N$ ) transmits its EB message, while the first term is the probability that the transmitted EB message is correctly received by the joining node. It can be shown, through simple algebraic manipulations, that the above probability takes its maximum value at $p_{E B}^{*}=1 / N$. Hence, hereafter we will assume $p_{E B}=p_{E B}^{*}=1 / N$.

According to the TSCH network formation algorithm, a node willing to join the network turns on its radio and listens for EB messages on a given channel frequency $f$. Since $T_{E B}$ (expressed in number of timeslots) and the number of available channels $N_{c}$ are assumed to be 
relatively prime, due to the channel hopping mechanism, EB messages are transmitted on different frequencies in subsequent cycles of duration $T_{E B}$ (throughout referred to as EB cycles, or cycles for short). Hence, at a given cycle, the joining node can receive an EB message only if the latter is transmitted on channel $f$ in that cycle.

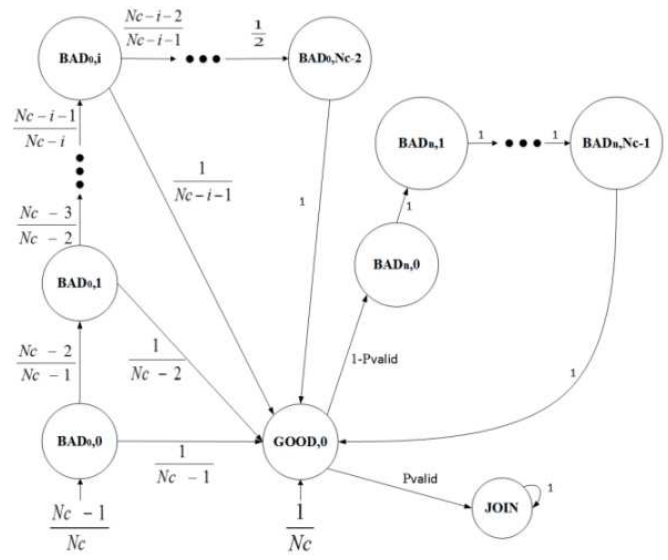

Fig. 2. Discrete Time Markov Chain.

We observe the state of the system at the beginning of each EB cycle (i.e., just before the transmission of the EB message). Let $B_{0}$ denote the EB cycle when the joining node starts listening for EB messages. We define an EB cycle as GOOD if node $u$ is using the same channel frequency as the advertiser nodes in that cycle, and $B A D$ otherwise. We also distinguish bad cycles as $\mathrm{BAD}_{0}$, if a good cycle has not been encountered yet, and $\mathrm{BAD}_{\mathrm{n}}$ otherwise. The state of node $u$ at the beginning of any EB cycle $B_{i}(i=0,1,2, \ldots)$ can be represented by means of a couple (cycle_state, seqnum), where cycle_state may be GOOD, $\mathrm{BAD}_{0}$, or $\mathrm{BAD}_{\mathrm{n}}$, while seqnum specifies the number of consecutive cycles already experienced in that state. We also consider a special state, referred to as JOIN where node $u$ moves as soon as it receives a valid EB.

Figure 2 shows how the state of node $u$ evolves over time. Let $N_{c}$ denote the number of channels available for communication. Assuming that the joining node has no information about the frequency used by the advertiser nodes, initially it starts either in state (GOOD,0), with probability $1 / N_{C}$, or in state $\left(\mathrm{BAD}_{0}, 0\right)$, with probability $\left(N_{c}-1\right) / N_{C}$

Assuming that the joining node is initially in state $\left(\mathrm{BAD}_{0}, 0\right)$, in the next step it will move to state (GOOD, 0$)$, if it encounters a good EB cycle, which occurs with probability $1 /\left(N_{c}-1\right)$, or to state $\left(\mathrm{BAD}_{0}, 1\right)$, if the next $\mathrm{EB}$ cycle is bad, which occurs with probability $\left(N_{c}-2\right) /\left(N_{c}-1\right)$. Similarly, let us assume that, at a given time, the joining node is in state $\left(\mathrm{BAD}_{0}, i\right)$, for $i=1,2, \ldots, N_{c}-3$. This means that it has already experienced $i$ consecutive bad cycles. Hence, in the next step it will move to state $(\mathrm{GOOD}, 0)$, if the next cycle is good, i.e., with probability $1 /\left(N_{c}-i-1\right)$, or to state $\left(\mathrm{BAD}_{0}, i+1\right)$ in case of another bad cycle, i.e., with probability $\left(N_{c}-i-2\right) /\left(N_{c}-i-1\right)$. If the joining node always experience bad cycles, after
$N_{c}-2$ steps it reaches state $\left(\mathrm{BAD}_{0}, N_{c}-2\right)$. However, the next cycle will be necessarily a good one, and the joining node will move to state (GOOD, 0).

Let us now consider the evolution from state (GOOD, 0 ). Since the EB cycle is good, the joining node has an opportunity to receive a valid $\mathrm{EB}$ message and complete the join, moving to the absorbing state JOIN, where it remains forever. According to Equation (2), the latter transition occurs with a probability $P_{\text {valid }}$. If the joining node misses the EB (i.e., with probability $\left.1-P_{\text {valid }}\right)$ it has to wait for more $N_{c}-1$ cycles to have a new opportunity. Hence, in Figure 2, the chain evolves through a sequence of states, each accessed with probability 1 , and finally reaches state (GOOD,0) again. This sequence models a deterministic delay equal to $\left(N_{c}-1\right)$ EB cycles.

The random process described so far, whose transition state diagram is depicted in Figure 2, is a discrete time Markov chain, as (i) state transitions occur at discrete times, and (ii) the probability to move to a new state only depends on the previous state. Its transition probability matrix $\boldsymbol{P}$ can be easily derived by using the transition probabilities shown in Figure 2. Through the transition probability matrix, we can derive the probability $P_{\text {join }}(k)$, i.e., the probability that the joining node receives a valid EB message, thus joining the network, after exactly $k$ EB cycles from $B_{0}$. To this end, let us denote by $\Omega$ the set of states of the Markov chain. We can sort states so that the initial states $(\mathrm{GOOD}, 0)$ and $\left(\mathrm{BAD}_{0}, 0\right)$ are the first one and the second one in the sequence, and the absorbing state JOIN is the last one in the sequence. Let $v_{0}$ denote the initial probability vector and $\boldsymbol{v}_{k}$ the probability vector after $k \mathrm{~EB}$ cycles from $B_{0} \quad(k=0,1,2, \ldots$.$) . Without losing in$ generality, we can assume

$$
\boldsymbol{v}_{0}=\left[1 / N_{C},\left(N_{c}-1\right) / N_{C}, 0, \ldots, 0\right]
$$

Hence, $\boldsymbol{v}_{k}=\boldsymbol{v}_{0} \cdot \boldsymbol{P}^{k}$. Let $|\Omega|$ denote the cardinality of $\Omega$, i.e. the total number of states. Since JOIN is the last state in the sequence (according to the selected order) it follows

$$
P_{\text {join }}(k)=v_{k}[|\Omega|]
$$

Let us indicate as $\mu_{(\mathrm{GOOD}, 0)}$ and $\mu_{(\mathrm{BAD}, 0)}$ the average time spent by the joining node $u$ to reach state JOIN when it starts from state $(\mathrm{GOOD}, 0)$ and $\left(\mathrm{BAD}_{0}, 0\right)$, respectively. The computation of $\mu_{i}, \forall i \in \Omega$, can be performed solving the following system of equations, with $\mu_{i}$ as unknowns:

$$
\mu_{i}=1+\sum_{j \in \Omega} p_{i j} \cdot \mu_{j}
$$

where $p_{i j}$ is the transition probability from state $i$ to state $j$.

Then, the average joining time $\tau_{\text {join }}$ can be derived as:

$$
\tau_{\text {join }}=\frac{1}{N_{c}} \cdot \mu_{(\mathrm{GOOD}, 0)}+\frac{\left(N_{c}-1\right)}{N_{c}} \cdot \mu_{(\mathrm{BAD}, 0)}
$$

\section{RESULTS}

In this section we evaluate the performance of the considered advertisement algorithm in terms of joining time. To this end, we use the analytical formulas derived in the previous section. We also use simulation to (i) validate our analytical model, and (ii) investigate the algorithm performance when the advertiser nodes use more than one channel offset to transmit their EB messages. 
To this end, we implemented the advertisement algorithm using the ns 2 simulation tool [22]. We modeled the same scenario considered in the analysis. However, in our simulation experiments we relaxed the assumption that all the advertiser nodes use the same channel offset to transmit their EB messages. Instead, channel offsets are deterministically assigned to advertiser nodes, so as to ensure a fair utilization of all channel offsets. Of course, the number of channel offsets (throughout referred to as $N_{o}$ ), must be less than, or equal to, the number of available channel frequencies $N_{c}$. When using more channel offsets, the number of (neighboring) advertiser nodes using the same channel offset reduces with $N_{o}$ (on average it is equal to $N / N_{o}$ ) Hence, the EB collision probability reduces accordingly. In our experiments $p_{E B}$ is set autonomously by each advertiser node, depending on the number of neighboring advertiser nodes using the same offset.

For each experiment we performed several independent replications. The simulation results shown below are averaged over all replications. We also derived confidence intervals by using the independent replications method and 95\% confidence level. However, in our results, confidence intervals are so small that they cannot be appreciated in the presented plots. We found that simulation results almost overlap analytical results in all the considered scenarios. The analytical results, that refer to the case when a single channel offset is used, represent an upper bound for the joining time experienced by connecting nodes. Also, the analytical formulas allows to better understand the behavior of the system.

In the following discussion, unless stated otherwise, we will assume that there are 3 advertiser nodes from which the joining node can potentially receive an EB message i.e., $(N=3)$, all channels are available for communications (i.e., $N_{c}=16$ ) and EB messages are never corrupted, i.e., the packet error rate $\left(p_{E R R}\right)$ is null. In our analysis we varied each of the above-mentioned parameters, one at a time, to analyze its influence on the performance of the advertisement algorithm.

Fig. 3 shows the average joining time expressed in number of $E B$ cycles (of duration $T_{E B}$ ), as a function of the number of advertiser nodes. We considered different numbers of channel offsets $\left(N_{o}\right)$ used by the advertiser nodes to broadcast their EB messages. As expected, the highest joining time is experienced when a single channel offset is used. In addition, in this case, the average joining time increases very quickly with the number of (neighboring) advertiser nodes. This is due to repeated collisions experienced in the transmissions of EB messages (see Equation (2)). Using more channel offsets reduces significantly the average joining time and, hence, the energy spent by devices to join the network. On the other side, it reduces the bandwidth available for data transmissions. Moreover, when using $N_{o}$ different channel offsets, on average, $N_{o}$ EB messages are transmitted instead of one - at each EB cycle, since advertiser nodes transmit EB messages with a probability $p_{E B}$ depending on the number of neighbors using the same channel offset.
Hence, the energy spent to advertise the network presence increases with $N_{o}$.

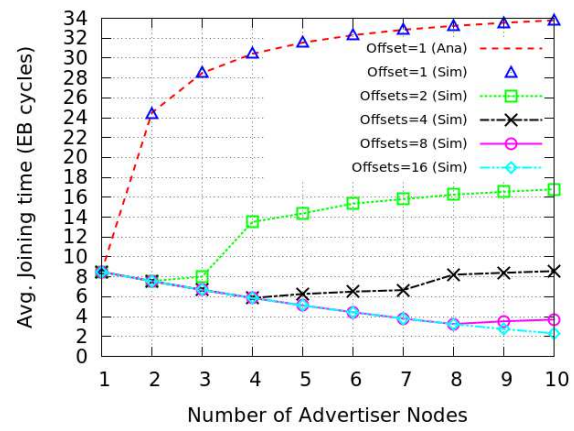

Fig. 3. Avg. joining time vs. \# of advertiser nodes $\left(\boldsymbol{N} \boldsymbol{c}=\mathbf{1 6}, \boldsymbol{p}_{E R \boldsymbol{R}}=\mathbf{0}\right)$.

Fig. 3 also shows that increasing the value of $N_{o}$ is beneficial only if the number of neighboring advertiser nodes is larger than the current number of channel offsets. For this reason, in Fig. 3, curves corresponding to different values of $N_{o}\left(N_{o} \geq 2\right)$ overlap in the first part. In conclusion, using many different channel offsets (e.g., more than 4) may be useful only when the network is very dense and, thus, there are many neighboring advertiser nodes.

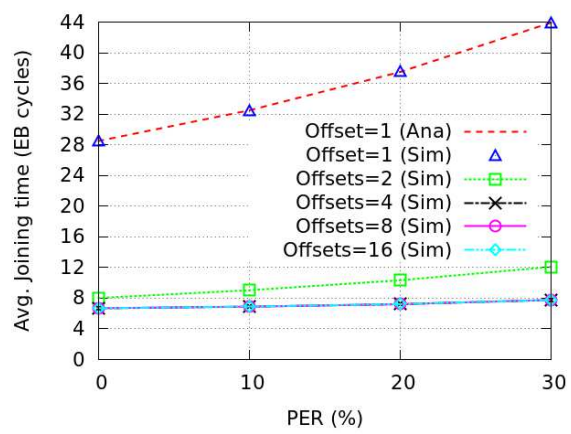

Fig. 4. Average joining time for different packet error rates $(\boldsymbol{N}=\mathbf{3}, \mathbf{N} \boldsymbol{c}=$ 16).

In the previous experiments we assumed that EBs are never corrupted by transmission errors. Of course, this is an optimistic assumption. Fig. 4 shows the influence of the packet error rate on the average joining time when there are 3 advertiser nodes from which the joining node can potentially receive an EB message $(N=3)$ and all channel frequencies are available $\left(N_{c}=16\right)$. As expected, the joining time increases as the wireless medium becomes more and more unreliable. The amount of this increase strongly depends on the number of channel offsets $N_{o}$.

When the packet error rate passes from $0 \%$ to $30 \%$, the joining time increases by about $55 \%$ with a single offset, while the increase is approximately $25 \%$ and $15 \%$ with 2 or more channel offsets. This is because (i) the percentage of non-valid EB transmissions, due to collisions, is higher when the number of channel offset is low (e.g., $N_{o}=1$, see Equation (2)) and (ii) the time a joining node has to wait, after each non-valid EB transmission, to have a new opportunity, decreases as $N_{o}$ increases. Hence, the effects of transmission errors are more severe when using a single channel offset. 


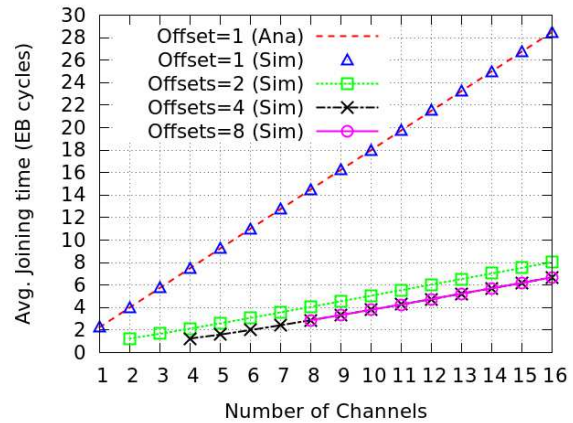

Fig. 5. Avg. joining time for a varying \# of available channels $(N=3)$.

So far we have assumed that all the available channel frequencies can be used for advertising EBs. In the next set of experiments we assume that only a subset of channel frequencies are used for this purpose (we also assume that joining nodes are aware of the subset of frequencies used for EB advertisement). Fig. 5 shows that, for any number of channel offsets, the average joining time increases linearly with the number of channel frequencies $N_{c}$ used for advertisement. Obviously, the number of channel offsets must be lower than, or equal to, the number of channel frequencies (for this reason some curves start from a given point in Fig. 5). The results show that, using only a subset of channel frequencies for advertisement would reduce significantly the joining time. However, joining nodes should be aware of the subset of frequencies that is used. Also, they should start listening on one of the frequencies in the subset.

\section{CONCLUSIONS}

In this paper we have investigated the network formation process in $802.15 .4 \mathrm{e}$ TSCH sensor/actuator networks. Since the standard does not specify any algorithm for advertising Enhanced Beacons in order to announce the network presence, we have defined a simple, random-based advertisement algorithm. We have analyzed the proposed algorithm in terms of joining time, by analysis and simulation. We have found that the joining time is mainly influenced by the number of channel offsets used for advertising Enhanced Beacons. Using more channel offsets reduces significantly the joining time and, hence, the energy spent by devices to join the network. On the other side, it increases the number of transmitted EBs and, hence, the energy spent by advertiser nodes. Moreover, using a large number of different channel offsets for advertisement may be beneficial, in terms of reduced joining time, only when the network density is extremely high. As a future work, we plan to extend the analytical model presented in this paper to consider the use of multiple channel offsets for advertising the network presence.

\section{REFERENCES}

[1] Alcaraz, C., Najera, P., Lopez, J., Roman, R.: Wireless senso networks and the internet of things: Do we need a complete integration? In: 1st International workshop on the security of The internet of Things (SecIoT), Tokyo, Japan, (2010)

[2] Akyildiz, I. F., Kasimoglu, I. H.: Wireless sensor and actor networks: research challenges. Ad hoc networks, 2(4), (2004)
[3] Willig, A.: Recent and emerging topics in wireless industrial communications: A selection. Industrial Informatics, IEEE Transactions on, (2008)

[4] Milenković, A., Otto, C., Jovanov, E.: Wireless sensor networks for personal health monitoring: Issues and an implementation. Computer communications, (2006)

[5] Miorandi, D., Uhlemann, E., Vitturi, S., Willig, A.: Guest Editorial: Special section on wireless technologies in factory and industrial automation, Industrial Informatics, IEEE Transactions on, (2007)

[6] Lemmon, M., Ling, Q., Sun, Y.: Overload management in sensoractuator networks used for spatially-distributed control systems. In: Proceedings of the 1st international conference on Embedded networked sensor systems, ACM, (2003)

[7] Sinopoli, B., Sharp, C., Schenato, L., Schaffert, S., Sastry, S. S.: Distributed control applications within sensor networks. In: Proceedings of the IEEE, (2003)

[8] Platt, G., Blyde, M., Curtin, S., Ward, J.: Distributed Wireless Sensor Networks and Industrial Control Systems---A New Partnership. In: Embedded Networked Sensors, 2005. EmNetS-II. The Second IEEE Workshop on, (2005)

[9] Low, K. S., Win, W. N. N., Er, M. J.: Wireless sensor networks for industrial environments. In: Computational Intelligence for Modelling, Control and Automation, 2005 and International Conference on Intelligent Agents, Web Technologies and Internet Commerce, International Conference on, (2005).

[10] H. Alemdar, C. Ersoy, Wireless sensor networks for healthcare: A survey, Computer Networks,(2010).

[11] J. Ko, C. Lu, M. Srivastava, J. Stankovic, A. Terzis, Matt Welsh, Wireless Sensor Networks for Healthcare, Proceedings of the IEEE, 2010

[12] IEEE Standard for Information technology, Part 15.4; Wireless Medium Access Control (MAC) and Physical Layer (PHY) Specifications for Low-Rate Wireless Personal Area Networks (LR-WPANs), IEEE Computer Society, (2006)

[13] ZigBee Alliance, The ZigBee Specification version 1.0 (2007)

[14] Wireless Medium Access Control (MAC) and Physical Layer (PHY) Specifications for Personal Area Networks (WPANs), IEEE Standard 802.15.1, (2005)

[15] HART Field Communication Protocol Specification. HART Communication Foundation Std., version 7.4, revised in 2012 [Online]. Available: http://www.hartcomm.org/, (2007)

[16] Wireless Systems for Industrial Automation: Process Control and Related Applications, International Society of Automation (ISA) Standard ISA-100.11a, (2009).

[17] IEEE std. 802.15.4e, Part. 15.4: Low-Rate Wireless Personal Area Networks (LR-WPANs) Amendament 1: MAC sublayer, IEEE Computer Society, (2012).

[18] Palattella, M. R., Accettura, N., Dohler, M., Grieco, L. A., Boggia, G.: Traffic Aware Scheduling Algorithm for reliable low-power multi-hop IEEE 802.15. 4e networks. In: Personal Indoor and Mobile Radio Communications (PIMRC), 2012 IEEE 23rd International Symposium on, IEEE (2012).

[19] Accettura, N., Palattella, M. R., Boggia, G., Grieco, L. A., Dohler, M.: Decentralized Traffic Aware Scheduling for multi-hop Low power Lossy Networks in the Internet of Things. In World of Wireless, Mobile and Multimedia Networks (WoWMoM), (2013).

[20] T. Watteyne, X. Vilajosana, B. Kerkez, F. Chraim, K. Weekly, Q. Wang, S. Glaser, K.Pister, OpenWSN: a Standards-based Lowpower Wireless Development Environment, Transactions on Emerging Telecommunications Technologies, (2012).

[21] De Guglielmo D., Anastasi G., Seghetti A., From IEEE 802.15.4 to IEEE 802.15.4e: a Step towards the Internet of Things, in Advances onto the Internet of Things, Series on Advances in Intelligent Systems and Computing.

[22] Network Simulator Ns2, http://www.isu.edu/nsnam/ns [Online]. 